Gut, 1972, 13, 759-762

\title{
The composition of hepatic and gallbladder bile in patients with gallstones
}

\author{
C. MACKAY, J. N. CROOK, D. C. SMITH, AND R. A. McAllister \\ From the University Department of Surgery, Western Infirmary, Glasgow
}

SUMMARY Bile specimens were obtained from 17 patients with gallstones and 21 patients with duodenal ulcer. The specimens were obtained from the former by needle aspiration of the gallbladder and common bile duct at operation and from the latter by duodenal intubation. The concentrations of bile salt, phospholipid, and cholesterol were measured. Gallbladder bile from gallstone patients contained significantly more cholesterol than did 'duodenal' bile from duodenal ulcer patients. Hepatic bile from gallstone patients contained significantly more cholesterol than did gallbladder bile from the same patients. When the data were plotted on triangular coordinates the relative composition lay within the zone of cholesterol solubility in all 21 ulcer patients. The relative composition of hepatic bile lay outside the zone of cholesterol solubility in five gallstone patients, at the limits of cholesterol solubility in a further three, and within the micellar zone in the remaining nine patients. This suggests that supersaturation of hepatic bile with cholesterol is not the sine qua non for the production of cholesterol gallstones.

Small and Rapo (1970) and Vlahcevic, Bell, and Swell (1970) have reported that cholesterol gallstones are associated with the production by the liver of bile which is supersaturated with cholesterol with respect to phospholipid and bile salt. Small studied American Indians, a population known to have a significantly higher incidence of gallstone disease than the general population of the United States of America (Comess, Bennett, and Burch, 1967). Vlahcevic et al (1970) studied a group of patients in the Veterans Administration Hospital, Richmond, Virginia, and it is known that there is a higher incidence of gallstone disease in North America than in Great Britain (Gross, 1929; Lieber, 1952). This paper presents data from patients resident in the United Kingdom showing that not all patients with cholesterol gallstones secrete hepatic bile which is supersaturated with cholesterol.

\section{Materials and Methods}

Seventeen patients (three males and 14 females) admitted to the Western Infirmary, Glasgow, for elective cholecystectomy for gallstone disease were studied. The ages ranged from 19 to 74 years, the mean being 47 years. Each patient had a functioning gallbladder demonstrated preoperatively by oral

Received for publication 17 August 1972. cholecystography. At operation specimens of gallbladder and hepatic bile were aspirated. After opening the abdomen a needle was inserted into the fundus of the gallbladder and the contents completely aspirated to prevent any error which might arise from sampling at different levels within the gallbladder due to the stratification effect described by Thureborn (1966) and also to prevent expressing gallbladder bile into the common duct from manipulation during cholecystectomy. At a later stage in the operation, usually after ligation of the cystic duct, a specimen of bile was obtained from the common bile duct, either by direct needle puncture or by aspirating through a catheter inserted via the cystic duct. Any sample of bile which was contaminated with blood or pus or consisted of white bile was excluded from the series. When the operation was completed the stones were removed from the gallbladder, washed, dried, and retained for chemical analysis.

Twenty-eight patients admitted for elective surgical treatment of duodenal ulcer were studied as controls. We have ethical reservations about aspirating bile from the gallbladder in patients who are undergoing non-biliary surgery and accordingly we obtained bile from these patients by duodenal intubation performed before operation. The patients were fasted overnight and a single-lumen radioopaque tube was passed into the stomach under radiological 
control. After emptying the stomach the tube was passed on into the second part of the duodenum the contents of which were aspirated and discarded. Cholecystokinin, kindly supplied by Boots Pure Drug Company, was then injected intravenously in a dose of 1 unit per kilogram body weight and bile was aspirated from the duodenal tube. It has been shown by Thistle and Schoenfield (1971) and Vlahcevic, Bell, Juttijudata, and Swell (1971) that bile obtained by this method is representative of gallbladder bile.

All specimens of bile were transferred as soon as possible to a refrigerator and stored at $-18^{\circ} \mathrm{C}$ until analysed.

\section{CHEMICAL ANALYSES}

After thawing, the samples were thoroughly mixed by vigorous shaking just before taking an aliquot for chemical analysis.

\section{CHOLESTEROL ESTIMATION}

Cholesterol was estimated using a modification of the serum method described by Sperry and Webb (1950). Bile was added to isopropanol to precipitate and extract the lipid. Cholesterol was then precipitated with digitonin and the precipitate dried with acetone/ether and ether. Glacial acetic acid and Liebermann Burchard reagent were subsequently added and the intensity of the blue colour was measured using a photoelectric colorimeter.

\section{PHOSPHOLIPID ESTIMATION}

Phospholipid was estimated using a modification of the methods of Fiske and Subbarow (1925) and Bartlett (1959). The specimens were digested with sulphuric acid before being cleared with hydrogen peroxide. This method estimates the total phosphorus content of the specimen of bile. However, when these values were compared with those obtained by analysis of a lipid solvent extract of bile the results were very similar confirming that almost all the phosphorus present in bile comes from phospholipid. Duplicate specimens were analysed and a correlation coefficient of 0.9988 was obtained.

\section{BILE SALTS}

The total bile salt concentration of the specimens was estimated by the method of Talalay (1960) as modified by Admirand and Small (1968).

\section{GALLSTONE ANALYSIS}

Where the gallstones were small a representative whole stone was analysed, but where they were large a representative cross section of a stone was studied. About $70 \mathrm{mg}$ of powdered stone was placed in a weighed centrifuge tube. The weight was then accurately determined and the volume made up to $10 \mathrm{ml}$ with chloroform-methanol $(2: 1 \mathrm{v} / \mathrm{v})$. The sample was then shaken repeatedly over several hours and then centrifuged. The supernatant was removed and the deposit dried to constant weight. A sample of the extract, usually 0.05 or $0.1 \mathrm{ml}$, was taken for cholesterol determination. From these measurements it was possible to determine the percentage of lipid, non-lipid, and cholesterol, present in each stone, or section of stone, and only patients whose gallstones were composed of at least $50 \%$ cholesterol were included in the study.

\section{Results}

The relative molar compositions were calculated by

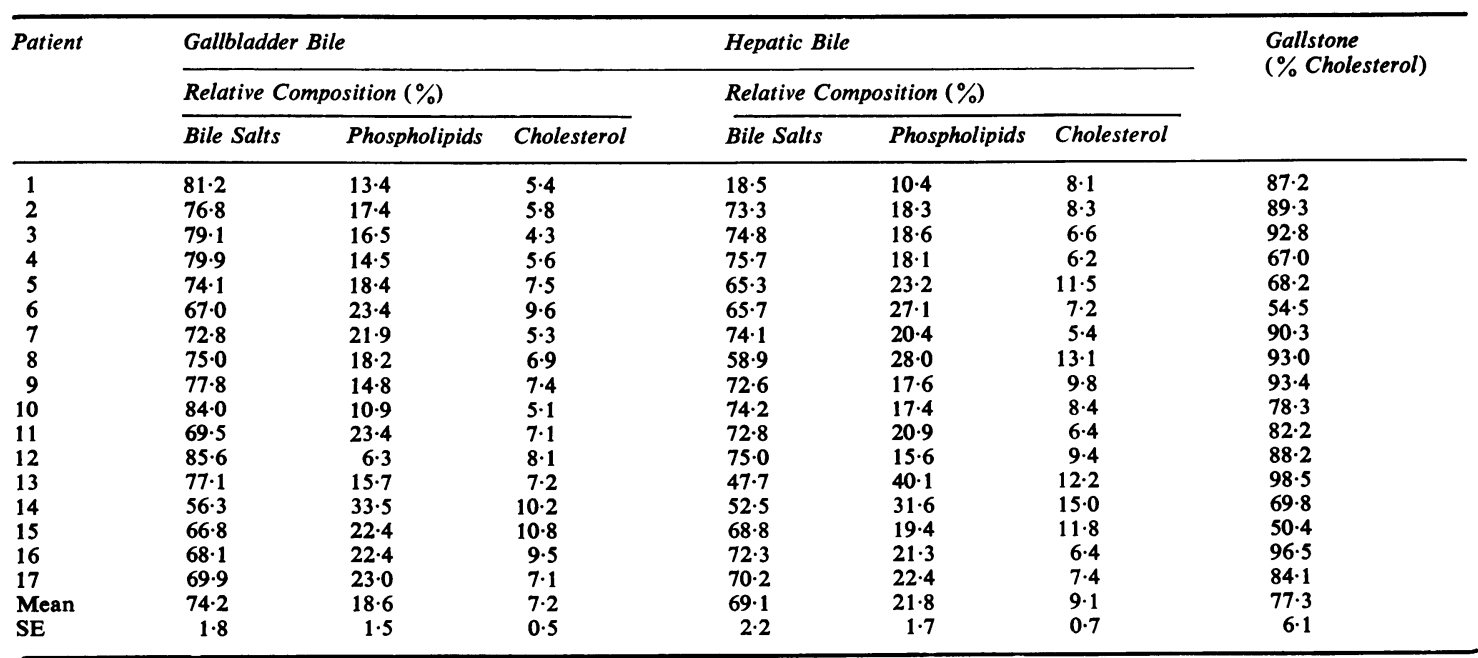

Table I Composition of bile and gallstones in patients with gallstones 
the technique of Admirand and Small (1968). Table I lists the composition of gallbladder and hepatic bile in the 17 patients with gallstones together with the cholesterol content of the gallstones. The mean totals of the three principal lipids, viz, bile salt, phospholipid, and cholesterol, in gallbladder and hepatic bile were 8.5 and $4.0 \mathrm{~g} / 100 \mathrm{ml}$ respectively. When these data are plotted on triangular coordinates (Fig. 1), as described by Admirand and Small (1968), it is seen that the relative composition of gallbladder bile lay outside the zone of cholesterol solubility in three patients, at the limits of cholesterol solubility in a further two patients, and within the micellar zone in the remaining twelve. Hepatic bile from these 17 patients lay outside the micellar zone in five (the sample from patient number 13 lies beyond the limits of the diagram), at the limits of cholesterol solubility in a further three, and within the micellar zone in the remaining nine patients.

In the 21 patients with duodenal ulcer the mean concentrations of the principal lipids in 'duodenal' bile were bile salt $58.4 \pm 6 \cdot 7$, phospholipid $13.2 \pm$ 1.9 , and cholesterol $3.8 \pm 0.5 \mathrm{~m}$ mole $/ 1$, and the mean relative composition was bile salt $76.1 \pm$ $1.7 \%$, phospholipid $18.7 \pm 1.5 \%$, and cholesterol $5.1 \pm 0.4 \%$. Figure 2 shows the relative composition of each of the 21 samples of duodenal bile plotted on triangular coordinates and it is evident that each lies within the zone of cholesterol solubility. The mean total of the three principal lipids in these 21 patients was $4.4 \mathrm{~g} / 100 \mathrm{ml}$.

When the relative composition of gallbladder bile

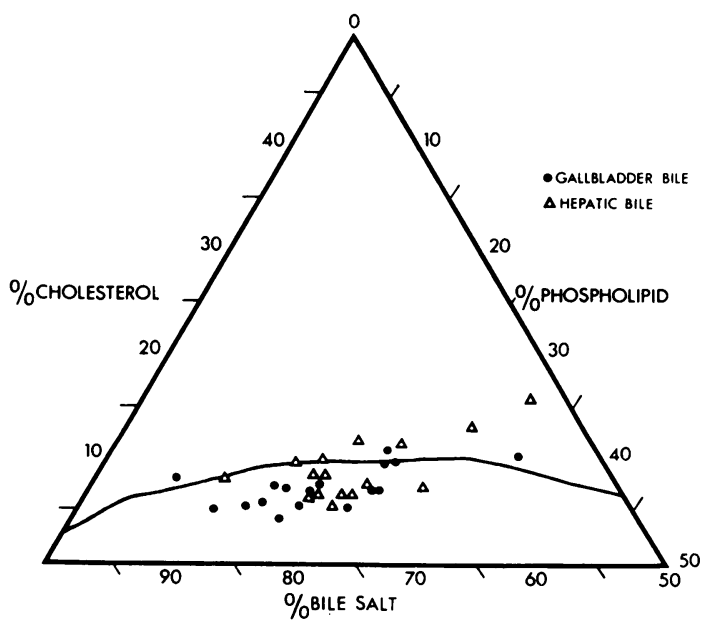

Fig. 1 Relative composition of gallbladder and hepatic bile in patients with gallstones. in gallstone patients is compared with the relative composition of 'duodenal' bile in duodenal ulcer patients the former is found to contain significantly more cholesterol $(7.2 \pm 0.5 \%$ and $5.1 \pm 0.4 \%$, $P<0.001$ using a standard $t$ test) but there is no significant difference between the relative amounts of bile salt $(74.2 \pm 1.8 \%$ and $76.1 \pm 1.7 \%)$ and phospholipid (18.6 $\pm 1.5 \%$ and $18.7 \pm 1.5 \%)$.

Comparison of the mean relative compositions of gallbladder and hepatic bile in gallstone patients reveals that the former contains significantly more bile salt $(74.2 \pm 1.8 \%$ and $69.1 \pm 2.2 \%, P<0.05$ using a paired $t$ test) and significantly less cholesterol $(7.2 \pm 0.5 \%$ and $9.1 \pm 0.7 \%, P<0.002)$ but there was no statistically significant difference in the relative amounts of phospholipid $(18.6 \pm 1.5 \%$ and $21 \cdot 8 \pm 1 \cdot 7 \%$ ).

\section{Discussion}

Admirand and Small (1968) have shown that the physical state of bile is determined by the relative amounts of bile salt, phospholipid, and cholesterol present. They showed that gallbladder bile from patients with gallstones was saturated with cholesterol whereas that from patients without gallstones was unsaturated. Small and Rapo (1970) and Vlahcevic et al (1970) then showed that in patients with gallstones the liver produced bile which was supersaturated with cholesterol and postulated that this was the primary aetiological factor in the production of cholesterol gallstones. The data presented here confirm that gallbladder bile from gallstone patients

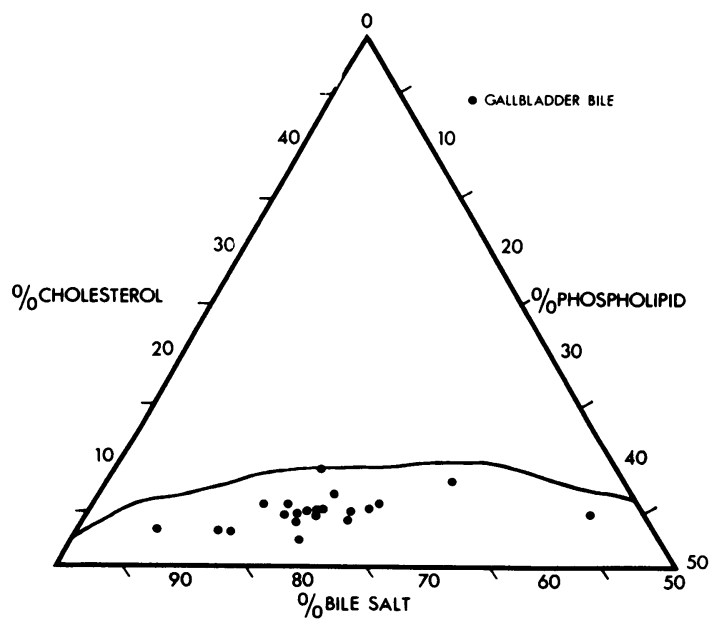

Fig. 2 Relative composition of gallbladder bile in patients with duodenal ulcer. 
contains significantly more cholesterol than does 'gallbladder bile' obtained by duodenal intubation from duodenal ulcer patients. We have also confirmed that in gallstone patients hepatic bile contains significantly more cholesterol than does gallbladder bile. We have not, however, been able to show that all patients with cholesterol gallstones produce hepatic bile which is supersaturated with cholesterol. Indeed in only five of our 17 gallstone patients was the relative composition of bile outside the zone of cholesterol solubility in the phase diagram. In another three patients the relative composition lay at the limits of cholesterol solubility whereas in the remaining nine patients bile was undersaturated with cholesterol. This would seem to suggest that while supersaturation of hepatic bile is one factor concerned with the production of cholesterol gallstones it is not the sole factor.

It would be interesting to compare the relative compositions of hepatic and gallbladder bile in patients without gallstone disease. This has not as yet been done because of the obvious difficulty of obtaining hepatic bile from such patients.

Other workers have reported information from gallstone patients showing that the hepatic bile is not always supersaturated with cholesterol. Burnett (1965) reported data on the composition of gallbladder and hepatic bile obtained at cholecystectomy and when these data were plotted on triangular coordinates by Small (1968) the relative composition of both lay within the micellar zone. Thureborn (1962) and Sarles, Crotte, Gerolami, Mule, Domingo, and Hauton (1971) have studied gallstone patients with indwelling T-tubes in the immediate post-cholecystectomy period. When Thureborn's data were plotted on triangular coordinates by Small (1968) the relative compositions of hepatic bile were found to lie within the zone of cholesterol solubility. Sarles et al (1971) studied the effect of diet on the composition of hepatic bile in four patients. Only when the patients were on a high calorie diet was there a tendency for the relative composition of bile to lie outside the zone of cholesterol solubility.

Bile supersaturated with cholesterol has also been found in patients with no evidence of gallstones. Nakayama and van der Linden (1970) studied gallbladder bile obtained at laparotomy from 20 patients with no evidence of hepatobiliary disease. In 12 of these 20 patients the relative composition lay outside the zone of cholesterol solubility. Vlahcevic et al (1970), describing the presence of supersaturated hepatic bile in patients with cholesterol gallstones, reported data from two patients without evidence of gallstones. In both these patients the relative composition of gallbladder and hepatic bile lay outside the micellar zone. They suggest that these patients may form gallstones in the future but this would seem to be begging the question.

It would seem therefore that cholesterol gallstones can occur in the presence of hepatic bile which is not saturated or supersaturated with cholesterol. There is always the possibility that supersaturation had been present some time in the past and had initiated gallstone formation. This criticism can be levelled at all studies of bile composition in patients with established gallstone disease. It may be that supersaturation of bile with cholesterol is only one factor involved in the formation of gallstones. Other factors such as the presence of mucus, infection, protein, or stasis in bile may render the mixed micelles unstable so resulting in the precipitation of cholesterol and the formation of gallstones.

\section{References}

Admirand, W. H., and Small, D. M. (1968). The physicochemical basis of cholesterol gallstone formation in man. J. clin. Invest., 47, 1043-1052.

Bartlett, G. R. (1959). Phosphorus assay in column chromatography. J. biol. Chem., 234, 466-468.

Burnett, W. (1965). The pathogenesis of gall-stones. In The Biliary System: A Symposium of the NATO Advanced Study Institute, Newcastle-upon-Tyne, 1963, edited by W. Taylor, pp. 601-618. Blackwell, Oxford. Davis, Philadelphia.

Comess, L. J., Bennett, P. H., and Burch, T. A. (1967). Clinical gallbladder disease in Pima Indians. New Engl. J. Med., 277, 894898.

Fiske, C. H., and Subbarow, Y.(1925). The colorimetric determination of phosphorus. J. biol. Chem., 66, 375-400.

Gross, D. M. B. (1929). A statistical study of cholelithiasis. J. Path. Bact., 32, 503-526.

Lieber, M. M. (1952). The incidence of gallstones and their correlation with other diseases. Ann. Surg., 135, 394-405.

Nakayama, F., and van der Linden, W. (1970). Bile from gallbladder harbouring gallstone: can it indicate stone formation? Acta chir. scand., 136, 605-610.

Sarles, H., Crotte, C., Gerolami, A., Mule, A., Domingo, N., and Hauton, J. (1971). The influence of calorie intake and of dietary protein on the bile lipids. Scand. J. Gastroent., 6, 189191.

Small, D. M. (1968). Gallstones. New Engl. J. Med., 279, 588-593.

Small, D. M., and Rapo, S. (1970). Source of abnormal bile in patients with cholesterol gallstones. New Engl. J. Med., 283, 53-57.

Sperry, W. M., and Webb, M. (1950). A revision of the SchoenheimerSperry method for cholesterol determination. J. biol. Chem., 187, 97-106.

Talalay, P. (1960). Enzymatic analysis of steroid hormone. Meth. biochem. Anal., 8, 119-143.

Thistle, J. L., and Schoenfield, L. J., (1971). Induced alterations in composition of bile of persons having cholelithiasis Gastroenterology, 61, 488-496.

Thureborn, E. (1962). Human hepatic bile. Acta chir. scand., Suppl., 303.

Thureborn, E. (1966). On the stratification of human bile and its importance for the solubility of cholesterol. Gastroenterology, 50, 775-780.

Vlahcevic, Z. R., Bell, C. C., Jr., Juttijudata, P., and Swell, L. (1971). Bile-rich duodenal fluid as an indicator of biliary lipid composition and its applicability to detection of lithogenic bile. Amer. J. dig. Dis., 16, 797-802.

Vlahcevic, Z. R., Bell, C. C., Jr., and Swell, L. (1970). Significance of the liver in the production of lithogenic bile in man. Gastroenterology, 59, 62-69. 